This is the author's accepted version of the manuscript for personal use. The definitive version is published by Nature Food at https://doi.org/10.1038/s43016-020-0132-8. Free to read version: https://rdcu.be/b6fbF

\title{
ORDER OF MEALS AT THE COUNTER AND DISTANCE BETWEEN OPTIONS AFFECT STUDENT CAFETERIA VEGETARIAN SALES
}

\section{ABSTRACT}

Altering the order in which meals are presented at cafeteria counters has been proposed as a way of lowering meat consumption, but remains largely untested. To address this, we undertook two experimental studies involving 105,143 meal selections in the cafeterias of a British university. Placing vegetarian options first on the counter consistently increased their sales when choices were widely separated $(>1.5 \mathrm{~m}$; vegetarian sales as a percentage of total meal sales increased by 4.6 and 6.2 percentage points) but there was no evidence of an effect when the options were close together $(<1.0 \mathrm{~m})$. This suggests that order effects depend on the physical distance between options.

KEY WORDS

Choice architecture; diet; climate change; vegetarian; order; position; nudge

\section{AUTHORS}

Emma E. Garnett ${ }^{\mathrm{a}, *}$, Theresa M. Marteau ${ }^{\mathrm{b}}$, Chris Sandbrook ${ }^{\mathrm{c}}$, Mark A. Pilling ${ }^{\mathrm{b}}$, Andrew Balmford ${ }^{\mathrm{a}}$

${ }^{a}$ Department of Zoology, University of Cambridge, CB2 3EJ, UK; ${ }^{b}$ Behaviour and Health Research Unit, University of Cambridge, Institute of Public Health, CB2 OSR, UK; ' Department of Geography, University of Cambridge, CB2 1QB, UK

* To whom correspondence may be addressed. Email: ee.garnett@gmail.com 
Shifting to more plant-based diets is a commonly proposed strategy to mitigate climate change and protect the natural environment ${ }^{1}$, particularly in high-income countries with high levels of animal product (meat, dairy, eggs, fish) consumption ${ }^{2}$. Traditional approaches to changing behaviour include information provision and taxation ${ }^{3}$. A third set of interventions - targeting non-conscious processes and the contexts in which behaviours occur, so-called "nudging" or "choice architecture" approaches - hold promise but are largely untested ${ }^{3}$. Rearranging the physical order in which foods are presented $-e . g$. in cafeteria lines - is widely advocated to achieve dietary change ${ }^{4}$, but the evidence for this is limited in both quantity and quality (see Supplementary Information (SI) Background) $)^{5,6}$. If effective, placing vegetarian options first would be a simple approach for reducing meat consumption. To our knowledge, our studies are the first to test the effect of order on vegetarian meal sales.

We worked in two college cafeterias (A and B) in the University of Cambridge (UK), using data on 105,143 meal selections across two academic years (Table 1). Experiments were run on week-day lunch and dinner times during university terms and involved alternating weekly between a vegetarian option ("VegFirst") and a meat option ("MeatFirst") being placed first in line, i.e. nearest the cafeteria entrance (Supplementary Figures S1-S4 and Supplementary Tables S1-S3). We hypothesised that the main-meal options placed first would be preferentially selected and therefore have higher sales. To better understand our initial results, we conducted a second study focused on the distance between choice options. In both studies we assessed the persistence of any effects detected through a follow-up monthly alternation of VegFirst and MeatFirst. We discuss results using binomial generalised linear models (GLMs) with order as the only predictor variable (univariate models) and when controlling for other predetermined independent variables (multivariate models, Table 1, SI Methods and Supplementary Tables S4-S9). 
Study 1

Study 2

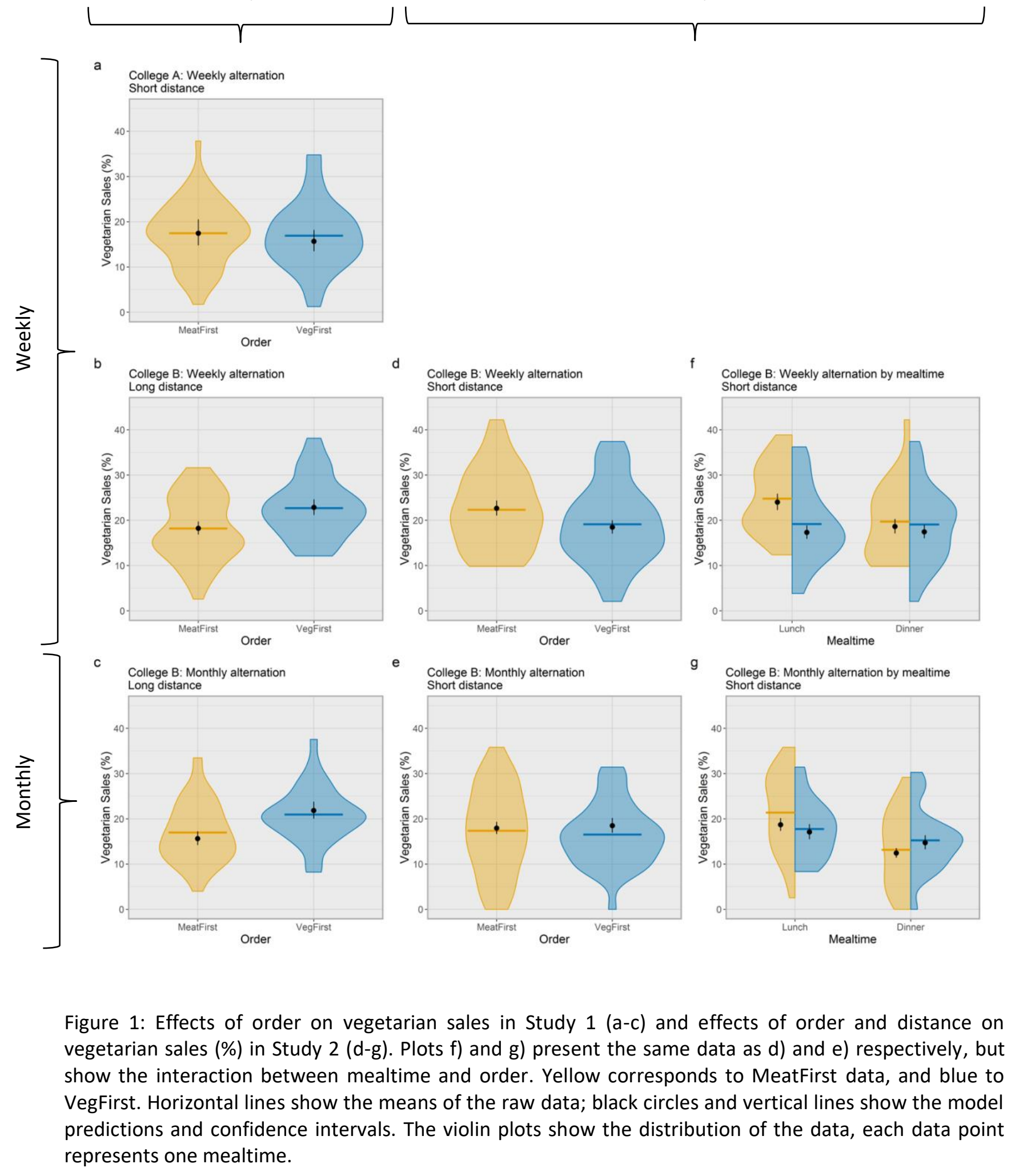

Figure 1: Effects of order on vegetarian sales in Study $1(a-c)$ and effects of order and distance on vegetarian sales (\%) in Study $2(\mathrm{~d}-\mathrm{g})$. Plots $\mathrm{f}$ ) and $\mathrm{g}$ ) present the same data as d) and e) respectively, but show the interaction between mealtime and order. Yellow corresponds to MeatFirst data, and blue to VegFirst. Horizontal lines show the means of the raw data; black circles and vertical lines show the model represents one mealtime. 
In Study 1, in College A, changing the order of meal options had no significant effect on vegetarian sales in either univariate $(p=0.876)$ or multivariate models $(p=0.058$; Figure $1 a$ and Table 1$)$. However, in College B, placing the vegetarian option first increased vegetarian sales by 4.6 percentage points $(25.2 \%$, Figure $1 \mathrm{~b})$ under the weekly alternation and by 6.2 percentage points (39.6\%) under monthly alternation. Meal order was a significant predictor of vegetarian sales $(p<0.001)$ in both univariate and multivariate analyses. The effect size of order was not significantly different between the weekly and monthly alternation i.e. the confidence intervals of the meal order odds ratio overlapped (Table 1), suggesting diners did not become habituated to order for at least one month after meal order was changed.

To summarise Study 1, we found no effects of altering meal order on vegetarian sales at College A, but strong and persistent effects at College B. We hypothesised that this result was due to the different distances between the vegetarian and meat options: $85 \mathrm{~cm}$ in College $A$ and $181 \mathrm{~cm}$ in College B. Previous studies have found that foods placed further away from participants are selected less frequently ${ }^{6}$, although to our knowledge no studies have tested interactions between distance and order. We designed Study 2 to test this hypothesis and rearranged College B's cafeteria to reduce the distance between the focal meal options to $67 \mathrm{~cm}$. The same protocols as in Study 1 were then implemented. Unfortunately increasing the separation of meal options in College A was not physically possible because of the cafeteria design.

In College B, Study 2, under the short-distance condition with weekly alternation of meal order, vegetarian sales were unexpectedly and significantly lower under VegFirst in both a univariate $(p<0.001)$ and multivariate model $(p<0.001 ;$ Table 1$)$. Further investigation of this result showed an interaction between mealtime and meal order ( GLM, interaction term $p<0.001)$ : at lunchtimes vegetarian sales were 6.7 percentage points $(29.7 \%)$ lower under VegFirst compared with MeatFirst, but there was no significant difference at dinnertimes. 
In the follow-up, monthly order alternation had no significant effect on vegetarian sales in the univariate analysis $(p=0.477)$ nor multivariate analysis ( $p=0.560$; Figure 1e). However, a significant interaction was again found between mealtime and meal order $(p<0.001)$ : there was no significant change in vegetarian sales with meal order at lunchtimes, but at dinner times vegetarian sales were 2.31 percentage points higher under VegFirst (Figure 1g). Overall, the results of Study 2 suggest that the effects of order did not persist in College B under short distance conditions, and are perhaps influenced by other aspects of the choice environment.

Our studies have several strengths. Firstly, they are based on 105,143 meal selections across two years. By contrast, a recent systematic review found a combined total of only 11,290 observations across 18 studies testing other choice architecture interventions to reduce meat consumption ${ }^{5}$. The current studies tested one intervention only, thus avoiding the confounding effects present in other studies on order ${ }^{6}$. By alternating the order of meals both weekly and monthly, the current studies were able to show that under the long-distance condition customers did not habituate to the effects of order for at least a month, which is obviously a key consideration in designing interventions for sustained effects. Follow-up experiments tested the inconsistent effects of order and established that the distance between options also influenced vegetarian sales. Finally, fidelity to protocol was high, estimated (from 76 observations) to be over $95 \%$ at both colleges.

These studies also have limitations. First, individual-level data on cafeteria visitors were not available to the researchers. This is common in field studies on food sales ${ }^{6}$ and means that there is some uncertainty in the p-value estimates. Second, the studies were conducted in British university cafeterias, a convenient but unrepresentative study setting. Studies in different populations other settings and countries are needed to test the generalisability of our results. Third, we cannot tell why the results in Study 2 did not replicate in the monthly alternation. Further studies will be needed to explicitly test for interactions and other influences on order (Supplementary Discussion). 
There are several possible mechanisms which might result in higher vegetarian sales under VegFirst when there is a longer ( $>1.5$ metre) distance between the vegetarian and meat options, but generally not under shorter distances $(<1$ metre). The distance between meal options might be a proxy for effort - generally, food options are chosen more frequently when less effort is required to obtain them ${ }^{6,7}$. A complementary hypothesis is that with increased distance the second option becomes less visible and salient than the first. Placing a vegetarian (instead of meat) meal on the counter, so that it was visible to restaurant customers at the point of meal selection, increased vegetarian sales in one study ${ }^{8}$. Further studies could test these mechanisms, and examine how effort and salience might interact with order (Supplementary Discussion).

To summarise, placing vegetarian options first consistently increased their relative sales when all options were widely separated, but not when close together. These findings have important implications for catering policies: a nudge which we predicted would increase vegetarian sales can work, but can also have no effect or even be counterproductive. For caterers interested in reducing meat sales, changing order - at least without pilot-testing its impacts - may be an unreliable and less effective strategy than alternative approaches such as increasing the relative availability of vegetarian options ${ }^{9}$ or reducing the serving sizes of meat ${ }^{5}$. Further studies are needed to specify more precisely the conditions under which placing vegetarian meals first increases the likelihood of their selection. 


\section{METHODS}

These studies consisted of multiple treatment reversal design experiments, swapping the order each week (or month) in which customers were presented with vegetarian and meat main-meal options at lunch and dinner times. The experiments were conducted in two University of Cambridge (UK) college cafeterias; a college is similar to a hall of residence or dorm. College A is a graduate college with over 600 students. College B has over 900 students, both undergraduate and graduate. Both colleges admit students of any gender identity. Meals are not included in the tuition or accommodation fees: students can choose to eat in the college cafeteria, cook their own meals or eat at other establishments. Students pay for meals by swiping their university cards. The cafeterias are largely self-service: students take a tray, view the different meal options available, and ask the serving staff for their preferred meal and side dishes. Students serve themselves salads, desserts and other cold items. These studies were approved by the University of Cambridge Psychology Research Ethics Committee (PRE.2016.100). Consent was obtained from catering managers; diners were not informed about the studies.

The primary outcome was the number of vegetarian meals sold at each mealtime, expressed as a percentage of the total meal sales. Salads, sandwiches and side dishes were not included. College A provided four options at lunch and five at dinner; sometimes a second vegetarian or VEGAN option was provided but this did not count towards the sales of the focal vegetarian option (SI Table S1). College B had a third main option, placed towards the back of the cafeteria. In summer term 2017 this third option was always meat at lunch and dinner (SI Table S2), but starting in autumn term 2017 at lunchtimes a vegan option was provided (SI Table S3). Similarly, the vegan sales did not contribute to the vegetarian sales considered in our analysis (see SI Tables S10 and S11). Following the recommendation of Simmons et $a l^{10}$ we discuss results from both univariate and multivariate models (SI Methods). 
Sales data were downloaded from the online catering platform Uniware ${ }^{11}$. Many individuals buy more than one meal from their college cafeteria over a term. In the absence of individual-level data, each meal selection was treated as independent. While this approach has been used in numerous other studies ${ }^{6}$, it adds uncertainty to p-value estimates. We therefore focused primarily on the effect size of our intervention, presenting the odds ratios and 95\% confidence intervals and McFadden's pseudo $\mathrm{R}^{2}$. The odds ratio - i.e. the effect size - was calculated by taking the exponential of the model estimate. Model diagnostics were used to check that the models did not violate any regression assumptions. We carried out all analyses in $\mathrm{R} 3.5^{12}$.

\section{DATA AVAILABILITY}

Data for the results in this paper can be found at https://doi.org/10.17863/CAM.41481

\section{CODE AVAILABILITY}

The code used for this analysis is available from the corresponding author on request.

\section{FUNDING}

This work was supported by E.G's NERC studentship grant number NE/L002507/1 and A.B.'s Royal Society Wolfson Research Merit award.

\section{ACKNOWLEDGEMENTS}

We thank Ivan, Danielle and lan for participating and generously contributing their time and cafeterias' data. We thank Georgia Stewart, Jonathan Williams and Josie Chambers for assisting with monitoring fidelity to protocol; Benno Simmons, Katie Saunders and Dominique-Laurent Couturier for assistance with analyses and coding in R, and Rachel Pechey, Emma Cartwright, Gareth Hollands and Milica Vasiljevic for their advice in setting up the experiment and interpreting our findings. We thank Kristian Nielsen for his feedback on drafts of this manuscript. 


\section{AUTHOR CONTRIBUTIONS}

E.E.G., A.B., C.S., and T.M.M. designed research; E.E.G. performed research; E.E.G. and M.A.P. analysed data; and E.E.G., A.B., C.S., and T.M.M. wrote the paper.

\section{REFERENCES}

1. Poore, J. \& Nemecek, T. Science 992, 987-992 (2018).

2. Bryngelsson, D. et al. Food Policy 59, 152-164 (2016).

3. Lehner, M. et al. J. Clean. Prod. 134, 166-177 (2016).

4. Thaler, R. \& Sunstein, C. Nudge. (Penguin, 2009).

5. $\quad$ Bianchi, F. et al. Lancet Planet Heal. 2, e384-e397 (2018).

6. Hollands, G. J. et al. Cochrane Database Syst. Rev. 9, CD012573 (2019).

7. Meiselman, H. et al. Appetite 23, 43-55 (1994).

8. Kurz, V. J. Environ. Econ. Manage. 90, 317-341 (2018).

9. Garnett, E. et al. Proc. Natl. Acad. Sci. 116, $20923-20929$ (2019).

10. Simmons, J. et al. Psychol. Sci. 22, 1359-1366 (2011).

11. Uniware. http://www.uniware.co.uk/ (2019)

12. R Core Team. https://www.r-project.org/ (2018). 
Table 1: Summary of experiments and multivariate model estimates for order in Studies 1 and 2. 95\% confidence intervals (Cls) reported.

\begin{tabular}{|c|c|c|c|c|c|c|c|c|c|c|c|}
\hline \multicolumn{7}{|c|}{ Study characteristics } & \multicolumn{5}{|c|}{ Multivariate model } \\
\hline Study & Term & College & $\begin{array}{l}\text { Distance } \\
\text { between } \\
\text { options } \\
(\mathrm{cm})\end{array}$ & $\begin{array}{l}\text { Order } \\
\text { alternation }\end{array}$ & $\begin{array}{l}\text { Number } \\
\text { of } \\
\text { mealtimes }\end{array}$ & $\begin{array}{l}\text { Number } \\
\text { of } \\
\text { meals }\end{array}$ & ${ }^{\mathrm{a}} \mathrm{R}^{2}$ & $\begin{array}{l}\text { b MeatFirst: Veg } \\
\text { sales \% [Cls] }\end{array}$ & $\begin{array}{l}{ }^{c} \text { VegFirst: Veg sales } \\
\%[\mathrm{Cls}]\end{array}$ & $\begin{array}{l}\text { d Meal order } \\
\text { odds ratio [Cls] }\end{array}$ & $\begin{array}{l}\text { e } \\
\text { order } \\
\text { value }\end{array}$ \\
\hline 1 & Spring 2017 & $A$ & $\begin{array}{l}\text { Short } \\
(85)\end{array}$ & Weekly & 92 & 11,683 & 0.084 & $17.5[14.8,20.5]$ & $15.7[13.5,18.2]$ & $\begin{array}{l}0.88 \quad[0.77, \\
1.00]\end{array}$ & 0.058 \\
\hline 1 & $\begin{array}{l}\text { Summer } \\
2017\end{array}$ & $B$ & $\begin{array}{l}\text { Long } \\
(181)\end{array}$ & Weekly & 96 & 20,544 & 0.070 & $18.2[16.8,19.7]$ & $22.8[21.2,24.6]$ & 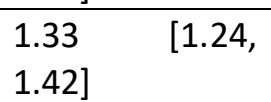 & $<0.001$ \\
\hline 1 & $\begin{array}{l}\text { Autumn } \\
2017\end{array}$ & B & $\begin{array}{l}\text { Long } \\
\text { (181) }\end{array}$ & Monthly & 86 & 22,518 & 0.111 & $15.6[14.2,17.2]$ & $21.8[20.0,23.8]$ & $\begin{array}{l}1.51 \quad[1.30, \\
1.75]\end{array}$ & $<0.001$ \\
\hline 2 & Spring 2018 & B & $\begin{array}{l}\text { Short } \\
(67)\end{array}$ & Weekly & 87 & 20,224 & 0.099 & $22.7[21.0,24.4]$ & $18.5[17.1,20.0]$ & $\begin{array}{l}0.77 \quad[0.72, \\
0.83]\end{array}$ & $<0.001$ \\
\hline “ & " & \multicolumn{3}{|c|}{ "Lunchtimes } & 45 & 10,236 & 0.115 & $24.0[22.3,25.9]$ & $17.3[15.9,18.8]$ & $\begin{array}{l}0.66 \quad[0.60, \\
0.73]\end{array}$ & $<0.001$ \\
\hline “ & " & \multicolumn{3}{|c|}{ " Dinnertimes } & 42 & 9,988 & " & $18.6[17.1,20.3]$ & $17.5[16.0,19.0]$ & $\begin{array}{l}0.92 \\
1.02]\end{array} \quad[0.83$, & 0.126 \\
\hline 2 & $\begin{array}{l}\text { Summer } \\
2018\end{array}$ & B & $\begin{array}{l}\text { Short } \\
\text { (67) }\end{array}$ & Monthly & 88 & 28,688 & 0.180 & $17.9[16.6,19.3]$ & $18.5[16.9,20.2]$ & $\begin{array}{ll}1.04 & {[0.92,} \\
1.18]\end{array}$ & 0.560 \\
\hline " & " & \multicolumn{3}{|c|}{ "Lunchtimes } & 45 & 14,177 & 0.189 & $18.7[17.3,20.2]$ & $17.1[15.5,18.8]$ & $\begin{array}{l}0.89 \quad[0.78, \\
1.03]\end{array}$ & 0.132 \\
\hline “ & " & \multicolumn{3}{|c|}{ Dinnertimes } & 43 & 14,511 & " & $12.4[11.4,13.5]$ & $14.7[13.3,16.4]$ & $\begin{array}{l}1.22 \\
1.40]\end{array} \quad[1.06$, & 0.007 \\
\hline
\end{tabular}

${ }^{a}$ McFadden's pseudo $\mathrm{R}^{2}$ for the multivariate model; ${ }^{\mathrm{b}}$ Model estimates for vegetarian sales (\% of total sales) under MeatFirst; 'Model estimates for vegetarian sales (\% of total sales) under VegFirst; ${ }^{d}$ Odds ratio for effect of VegFirst compared to MeatFirst (the reference category); ${ }^{e}$ Meal order variable $p$-value in multivariate model; ${ }^{\mathrm{f}}$ Model estimates for vegetarian sales at mealtimes from the multivariate model with an interaction between order and mealtime; the same model was run twice, once with Lunch-MeatFirst and once with Dinner-MeatFirst as the reference categories in order to generate odds ratios for both.

Independent variables included in multivariate models: mealtime, ambient temperature (centigrade), days since the start of the experiment, day of the week. Variables in College A only (as invariant in College B): vegetarian price differential, menu rotation, presence of an additional vegetarian option (Supplementary 
Methods) 